\title{
Successfully Treated Complete Atrioventricular Block with Corticosteroid in a Patient with Cardiac Sarcoidosis: Usefulness of Gallium-67 and Thallium-201 Scintigraphy
}

\author{
Ken Umetani, Tsukasa Ishinara, Kimio Yamamoto, Takao Sawanobori, Isao Kohno, Hiroshi IJiri, \\ Sadayoshi Komori and Kohji TAMURA
}

\begin{abstract}
A 71-year-old male with sarcoidosis was followed for 6 years without steroid therapy. He was admitted because of complete atrioventricular block. Chest X-ray showed hilar lymphadenopathy. Echocardiogram showed mild left ventricular hypertrophy without local wall thinning. Cardiac sarcoidosis was diagnosed by a defect of Thallium-201 (Tl201) imaging and abnormal uptake of Gallium-67 (Ga-67). After the start of corticosteroid therapy, complete atrioventricular block was recovered. Abnormal uptake of Ga67 was improved. Tl-201 and Ga-67 are useful to diagnose cardiac sarcoidosis, to evaluate the lesion of cardiac involvement and to estimate the efficacy of corticosteroid therapy. (Internal Medicine 39: 245-248, 2000)
\end{abstract}

Key words: cardiac scintigraphy, conduction block, systemic sarcoidosis

\section{Introduction}

Sarcoidosis is a systemic disorder of unknown cause $(1,2)$. The granulomatous process involves any organ, including lungs, heart, skin, eyes, the nervous system and kidneys, which deteriorate slowly over more than 10 years. Common presentation of cardiac sarcoidosis is cardiac rhythm disturbances, conduction block and cardiac pump failure (1-6). In Japan, the majority of deaths related to sarcoidosis has been reported to be due to cardiac sarcoidosis, probably due to fatal complete heart block or ventricular tachyarrhythmia $(7,8)$. However, to evaluate the activity of cardiac sarcoidosis and to predict the occurrence of atrioventricular block is very difficult as occult granulomatous lesions may exist in the heart (6). We treated a case with cardiac sarcoidosis with complete atrioventricular block which occurred after a long follow-up without corticosteroid therapy, and corticosteroid therapy was extremely effective to recover from atrioventricular block. In addition, Gallium-67 (Ga-67) and Thallium-201 (Tl-201) scintigraphy were useful to evaluate the activity of cardiac sarcoidosis.

\section{Case Report}

Six years previously, a 65 -year-old male patient with a diagnosis of uveitis, who felt easily fatigued for the previous three months, was referred to us to evaluate underlying disease. He had a history of left artificial pneumothorax at 21 years of age for the therapy of pulmonary tuberculosis, nephritis at 7 years of age and hypertension for the previous 14 years. The serum level of angiotensin-converting enzyme (ACE) was $26.4 \mathrm{IU} / \mathrm{l}$ (normal range 7.7-29.4 IU/l) and the serum creatinine level was slightly increased $(1.2 \mathrm{mg} / \mathrm{dl}$; normal range 0.5 $1.0 \mathrm{mg} / \mathrm{dl}$ ). Other laboratory findings were normal. A chest Xray showed right side hilar lymphadenopathy and left side pleural thickening (Fig. 1). There was no reticulonodularity in either lung field. An electrocardiogram (ECG) showed left ventricular hypertrophy without repolarization abnormalities (Fig. 2A). Percent vital capacity (\%VC) was $43.9 \%$ and forced expiratory volume in one second (FEV1\%) was $86.9 \%$. Ga-67 imaging showed bilateral hilar uptake with no uptake at the heart area. Echocardiogram (UCG) showed mild left ventricular hypertrophy with normal left ventricular function; intraventricular septal thickness (IVS) was $13 \mathrm{~mm}$ and left ventricular posterior thickness (LVPW) was $10 \mathrm{~mm}$, and other findings were normal. The ratio of $\mathrm{CD} 4 / \mathrm{CD} 8$ cells in bronchoalveolar lavage fluid was 0.9 (normal range $0.4-1.8$ ). Pathological findings of scalene lymph node biopsy showed noncaseating granuloma. Therefore, we diagnosed sarcoidosis without disordered function of vital organs. The patient was discharged and followed carefully without corticosteroid therapy.

He was readmitted to our hospital at 69 years of age (two years previously) complaining of palpitation which continued

From the Second Department of Internal Medicine, Yamanashi Medical University, Yamanashi

Received for publication February 3, 1999; Accepted for publication October 9, 1999

Reprint requests should be addressed to Dr. Ken Umetani, the Second Department of Internal Medicine, Yamanashi Medical University, 1110 Shimokato, Tamaho-machi, Nakakoma-gun, Yamanashi 409-3898 


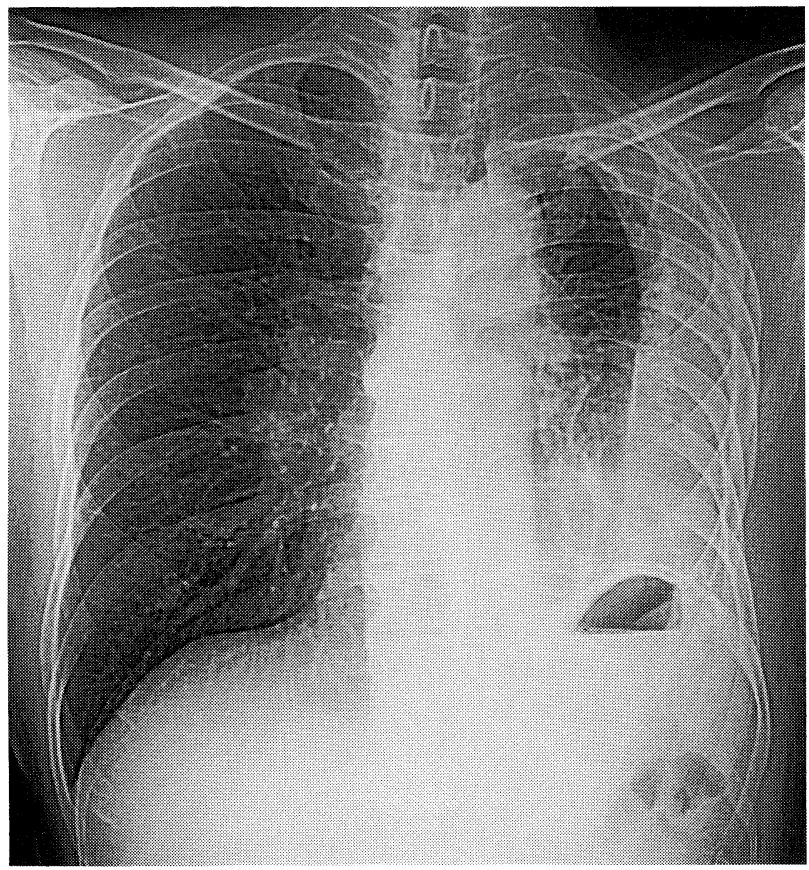

Figure 1. Chest X-ray taken on first admission. Right hilar lymph node adenopathy and left pleural thickening are observed.

for two to three hours. ECG described atrial flutter of 2:1 to 4:1 conduction. Atrial flutter was converted to sinus rhythm by disopyramide. 12-lead ECG and UCG findings were similar to those of 4 years previously. Holter ECG recording showed paroxysmal atrial flutter/fibrillation continuing for 30 minutes. Other than this arrhythmia, the basic rhythm was sinus without atrioventricular block. ACE was less than 1.0 IU/l. Although Ga-67 imaging showed an abnormal uptake in the right hilar lymph nodes, there was no abnormal uptake in the lung fields or heart area. He was followed taking disopyramide and no corticosteroid.

At 71 years of age, he complained of easy fatigue and bradycardia was pointed out. He was again admitted to our hospital, and was diagnosed with complete atrioventricular block with bradycardia $(\mathrm{HR}=38 \mathrm{bpm})$ (Fig. $2 \mathrm{~B})$. The chest $\mathrm{X}$-ray was almost the same as that of 6 years ealier. UCG showed mild left ventricular hypertrophy without segmental dyskinesis. An immediate temporary pacemaker was required. ACE was 3.3 IU/l. Six days later, cardiac catheterization and electrophysiological study were carried out. Coronary arteriography and left ventriculography was normal. Electrophysiological study showed A-H block. Endomyocardial biopsy from the right ventricular septum did not show the evidence of sarcoidosis. A permanent pacemaker was implanted (VVI pacing; rate 70 bpm). Ga-67 imaging showed an abnormal uptake at the heart and right hilar area in the absence of uptake in lung fields (Fig. 3A). In addition, Tl-201 (Fig. 4) revealed a reduced uptake at the basal region of the anteroseptal and inferior wall of the left ventricle. We could not confirm pathohistological evidence of
A: May 1995
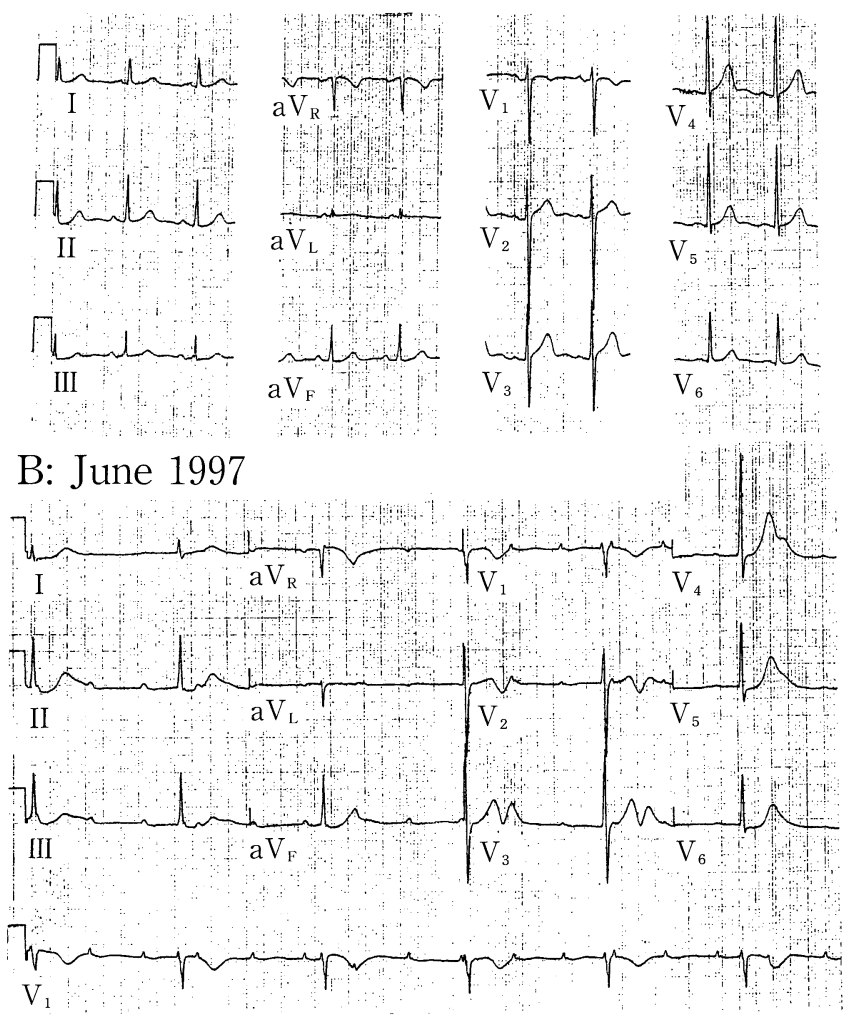

Figure 2. A: Electrocardiogram recorded on first admission (1991). There was no conduction block or repolarization abnormality. B: Electrocardiogram recorded when the patient was admitted for complete atrioventricular block (1997). The QRS-wave forms were almost the same as those of sinus rhythm except for a slight difference in QRS axis, which indicates that the escape beats originated from above or around His bundle. Ventricular rate is 36 bpm without intraventricular conduction delay.
A: Before

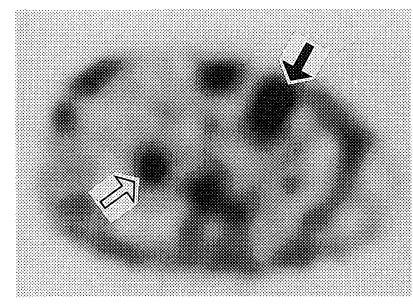

June 1997
B: After

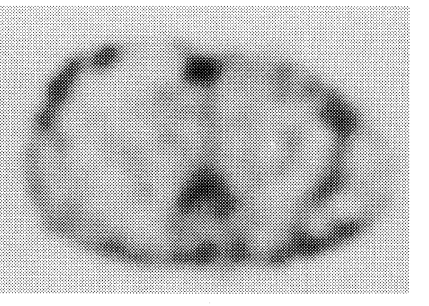

July 1997
Figure 3. Ga-67 imaging before (A) and after (B) corticosteroid therapy. The abnormal uptake at the heart (black arrow) and right hilar area (white arrow) disappeared after therapy. 
Short Axis

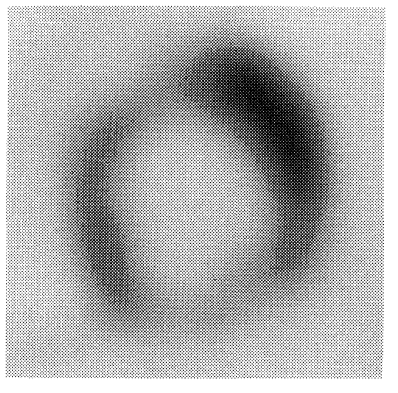

Vertical Axis

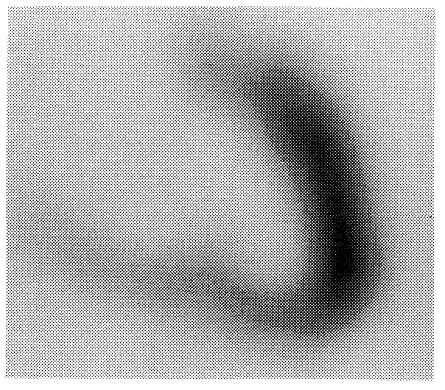

Figure 4. Tl-201 imaging before corticosteroid therapy. Defects of Tl-201 are noted at the base of anteroseptum and inferior to the left ventricle.

cardiac sarcoidosis. However, we confirmed pathohistological evidence of sarcoidosis 6 years previously, which indicates this complete AV block was due to cardiac sarcoidosis. Corticosteroid (prednisolone $30 \mathrm{mg} /$ day) and antituberculosis medication (Isoniazid $200 \mathrm{mg} /$ day and Rifampicin $300 \mathrm{mg} /$ day) were administered due to the past history of lung tuberculosis. On the 7th day after the start of corticosteroid therapy, atrioventricular conduction block was not observed in 12-lead ECG (PQ interval was $180 \mathrm{~ms}$ ). Holter ECG recording performed on the 14th day after the start of corticosteroid therapy showed no atrioventricular block. However, as ventricular pacing was programmed at $70 \mathrm{bpm}$, almost $50 \%$ of total QRS beats were pacing beats. Ga-67 imaging taken one month after the start of corticosteroid therapy showed no uptake at the heart and right hilar lymph node area (Fig. 3B). He was discharged and has been followed. Holter ECG recording performed 2 months after discharge also showed no atrioventricular block. At this time the pacemaker was programmed at $50 \mathrm{bpm}$, and only $2 \%$ of total QRS beats were pacing beats. 12-lead ECG recorded 5 months after discharge still showed sinus rhythm with normal atrioventricular conduction ( $\mathrm{PQ}=190 \mathrm{~ms})$. Tl-201 taken one years after the start of corticosteroid therapy showed no remarkable change compared to that taken before corticosteroid therapy.

\section{Discussion}

Cardiac sarcoidosis has been diagnosed in $27 \%$ of consecutive autopsied patients with systemic sarcoidosis in United States (6). In Japan, $52 \%$ of patients with systemic sarcoidosis died due to cardiac sarcoidosis in autopsied cases (8). Some patients with cardiac sarcoidosis die suddenly. The major cause of sudden death is due to conduction block or ventricular tachyarrhythmia. Numao (8) reported that $33 \%$ of fatal myocardial sarcoidosis patients suffered from third degree atrioventricular block and 36\% died suddenly. Therefore, it is very important to detect cardiac involvement, especially in Japanese patients. Suzuki et al (9) reported that the incidence of ventricular arrhythmia recorded by Holter ECG monitoring can predict the cardiac involvement of sarcoidosis, however the sensitivity and specificity are still low. These facts suggest difficulties in the diagnosis of cardiac involvement of sarcoidosis, particularly in the early stage of disease. The present case also indicates the difficulties in the prediction of the occurrence of atrioventricular block because there were no prior ECG changes or clinical symptom before the occurrence of atrioventricular block; only increased frequency of atrial tachyarrhythmia, paroxysmal atrial fibrillation and atrial flutter, may predict the cardiac involvement of sarcoidosis.

An interesting point of the present case is the characteristic imaging of Ga-67 and Tl-201 scintigraphy indicating cardiac involvement of sarcoidosis. ACE was within the normal range and UCG showed only mild left ventricular hypertrophy without focal ventricular wall thinning throughout the follow-up period, which could not indicate cardiac involvement of sarcoidosis. Tawarahara et al (10) and Okayama et al (11) reported that myocardial Tl-201 defects are useful to detect cardiac sarcoidosis and to evaluate the lesion size and location of cardiac involvement and that $\mathrm{Ga}-67$ uptake may predict the response to corticosteroid. The present case is consistent with their reports. A defect of Tl-201 scintigraphy localized in the basal part of the left ventricle anterior and inferior indicates the localized cardiac involvement in this region, although most of the previous cases had a patchy defect of the left ventricle. In addition, although uptake of Ga-67 disappeared after therapy (Fig. 3B), Tl-201 showed no remarkable change even after therapy. It was not clear whether the identification of the location between the defect of Tl-201 scintigraphy and the uptake of Ga-67. The lesion of defect of Tl-201 may be scarred area.

Ga-67-uptake at the heart was probably at the left ventricle (Fig. 3). However, echocardiography and left ventriculography showed normal wall motion. Abnormal Ga-67-scintigraphy may indicate an early stage of inflammation without abnormal wall motion. Actually the abnormal uptake of Ga-67 was reversible. In addition, electrophysiological study showed A-H block and escape beats were generated around His bundle during atrioventricular block. Ga-67 scintigraphy could not detect the abnormality of this lesion. Most myocardial infiltration of cardiac sarcoidosis is only histological small lesions (6). In this case, the slight infiltration, which can not be detected by Ga- 67 scintigraphy, and the constant defect of Tl201 at basal area, which may be scar lesion (Fig. 4), may have caused the reversible atrioventricular block.

Tl-201 and Ga-67 scintigraphy have significant diagnostic and prognostic value in cardiac sarcoidosis. Especially the change in Ga-67 uptake showed representative imaging to evaluate the effect of corticosteroid therapy.

\section{References}

1) Newman LS, Rose CS, Mainer LA. Sarcoidosis. N Engl J Med 336: 1224 1234, 1997.

2) Sharma OP, Maheshwari A, Thaker K. Myocardial sarcoidosis. Chest 103: 253-258, 1993 


\section{UMETANI et al}

3) Winters SL, Cohen M, Greenberg S, et al. Sustained ventricular tachycardia associated with sarcoidosis: Assessment of the underlying cardiac anatomy and the prospective utility of programmed ventricular stimulation, drug therapy and an implantable antitachycardia device. J Am Coll Cardiol 18: 937-943, 1991.

4) Shiotani H, Miyazaki T, Matsunaga K, Kado T. Improvement of severe heart failure with corticosteroid therapy in a patient with myocardial sarcoidosis. Jpn Circ J 55: 393-396, 1991.

5) Bajaj AK, Kopelman HA, Echt DS. Cardiac sarcoidosis with sudden death: Treatment with the automatic implantable cardioverter defibrillator. Am Heart J 116: 557-560, 1988.

6) Silverman KJ, Hutchins GM, Bulkley BH. Cardiac sarcoid: A clinicopathologic study of 84 unselected patients with systemic sarcoidosis. Circulation 58: 1204-1211, 1978.

7) Matsui $Y$, Iwai K, Tachibana T, et al. Clinicopathological study on fatal myocardial sarcoidosis. Ann NY Acad Sci USA 278: 455-469, 1975.

8) Numao Y. On the cardiac disorder of sarcoidosis; Multidimensional analysis of the author's 12 cases and a nationwide study in Japan with special reference to electrocardiographic diagnosis and treatment. Tokyo Women's Med Coll J 51: 2001-2031, 1981 (in Japanese).

9) Suzuki T, Kanda T, Kubota S, Imai S, Murata K. Holter monitoring as a noninvasive indicator of cardiac involvement in sarcoidosis. Chest 106: 1021-1024, 1994 (see comments).

10) Tawarahara K, Kurata C, Okayama K, Kobayashi A, Yamazaki N. Tha!lium-201 and gallium 67 single photon emission computed tomographic imaging in cardiac sarcoidosis. Am Heart J 124: 1383-1384, 1992.

11) Okayama K, Kurata C, Tawarahara K, Wakabayashi Y, Chida K, Sato A. Diagnostic and prognostic value of myocardial Scintigraphy with Thallium-201 and Gallium-67 in cardiac sarcoidosis. Chest 107: 330-334, 1995. 